\title{
A SURVEY OF CORONAL CAVITY DENSITY PROFILES
}

\author{
J. FULLER ${ }^{1,2}$ AND S. E. Gibson ${ }^{2}$ \\ ${ }^{1}$ Cornell University, 610 Space Sciences Building, Ithaca, NY 14853, USA \\ ${ }^{2}$ HAO/NCAR, P.O. Box 3000, Boulder, CO 80307-3000, USA \\ Received 2009 February 12; accepted 2009 June 1; published 2009 July 10
}

\begin{abstract}
Coronal cavities are common features of the solar corona that appear as darkened regions at the base of coronal helmet streamers in coronagraph images. Their darkened appearance indicates that they are regions of lowered density embedded within the comparatively higher density helmet streamer. Despite interfering projection effects of the surrounding helmet streamer (which we refer to as the cavity rim), Fuller et al. have shown that under certain conditions it is possible to use a Van de Hulst inversion of white-light polarized brightness $(p B)$ data to calculate the electron density of both the cavity and cavity rim plasma. In this article, we apply minor modifications to the methods of Fuller et al. in order to improve the accuracy and versatility of the inversion process, and use the new methods to calculate density profiles for both the cavity and cavity rim in 24 cavity systems. We also examine trends in cavity morphology and how departures from the model geometry affect our density calculations. The density calculations reveal that in all 24 cases the cavity plasma has a flatter density profile than the plasma of the cavity rim, meaning that the cavity has a larger density depletion at low altitudes than it does at high altitudes. We find that the mean cavity density is over four times greater than that of a coronal hole at an altitude of $1.2 R_{\odot}$ and that every cavity in the sample is over twice as dense as a coronal hole at this altitude. Furthermore, we find that different cavity systems near solar maximum span a greater range in density at $1.2 R_{\odot}$ than do cavity systems near solar minimum, with a slight trend toward higher densities for systems nearer to solar maximum. Finally, we found no significant correlation of cavity density properties with cavity height—indeed, cavities show remarkably similar density depletions - except for the two smallest cavities that show significantly greater depletion.
\end{abstract}

Key words: Sun: corona - Sun: coronal mass ejections (CMEs) - Sun: magnetic fields

Online-only material: color figures

\section{INTRODUCTION}

The genesis of solar coronal mass ejections (CMEs) is both an intellectually intriguing, fundamental unsolved problem in plasma astrophysics, and a societally relevant subject critical to space weather prediction and mitigation. Understanding the preeruption state of the corona is a top priority for space science. A common and compelling feature of white-light CMEs is their three-part morphology of a bright expanding loop, followed by a relatively dark cavity, and lastly a bright core associated with an erupting prominence (Illing \& Hundhausen 1986). It is also quite common for a prominence with a dark, semi-circular or circular cavity surrounding it to exist quiescently in the corona prior to the eruption. Multiple cases have been documented where such a quiescent cavity and its enclosed prominence bodily erupt as CMEs (Fisher \& Poland 1981; Hundhausen. 1999; Yurchyshyn 2002; Maricic et al. 2004; Sterling \& Moore 2004a; Vrsnak et al. 2004; Gibson et al. 2006). Coronal prominence cavities are thus clues to the state of the corona just prior to a CME, and may in fact be a signature of stored magnetic energy capable of driving the CME.

It is therefore critical to obtain information about cavity plasma density, temperature, velocity, and ideally magnetic field, via multi-wavelength observations. Cavities have been observed in white light (Gibson et al. 2006), radio (Marqué et al. 2002; Marqué 2004), soft X-ray, and extreme ultraviolet (Hudson et al. 1999; Hudson \& Schwenn 2000; Sterling \& Moore 2004b; Heinzel et al. 2008). Although these studies have provided some information about cavity density and temperature, many questions remain unanswered. The questions of whether the cavity is hotter or cooler than the surrounding helmet streamer, and of how cavity density depletion varies within the cavity are examples of important constraints that need to be observationally established to properly constrain coronal cavity models (Zhang \& Low 2004; van Ballegooijen $\&$ Cranmer 2008; Fuller et al. 2008).

In this paper, we focus on what can be learned about cavities from white-light observations, in particular regarding the distribution of density in cavities relative to their surrounding helmet streamer (which we refer to as the "cavity rim"). We will use data from the Mauna Loa Solar Observatory Mark 4 (Mk4) white-light coronameter, which has a field of view from about 1.14 to 2.86 solar radii and a 3 minute observing cadence. The Mk4 coronameter measures polarized brightness $(p B)$, which is a measure of white light scattered off of coronal electrons. The quantification of coronal density within a cavity from $p B$ is complicated by projection of intervening features along the line of sight. Because the white-light corona is optically thin, each pixel in a coronagraph image contains contributions from light scattered off electrons along the entire line of sight. Consequently, the brightness of a pixel in a coronagraph image of a cavity contains contributions from both the cavity material and the surrounding helmet streamer. A Van de Hulst inversion of polarized brightness data requires the cylindrical symmetry of material along the line of sight (Van de Hulst 1950), a symmetry that is broken if a pixel contains contributions from materials of differing density profiles (such as the cavity and cavity rim).

However, if we know the locations at which material with differing density profiles lies along the line of sight, we can apply correcting factors to the $p B$ data that allow for a Van de Hulst inversion to calculate the density of cavity or cavity rim material. In the method used by Fuller et al. (2008), the cavity is 


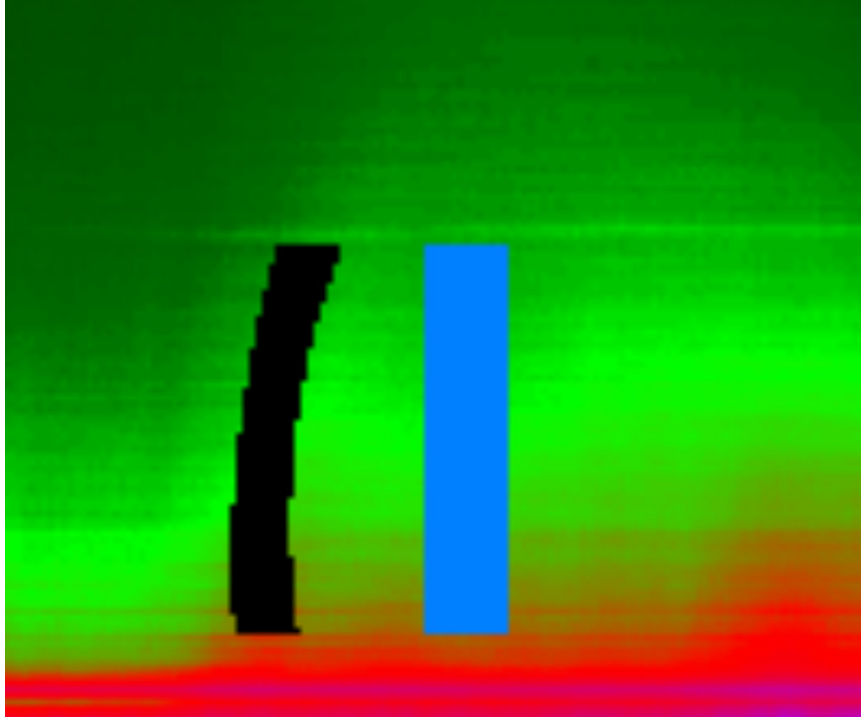

Figure 1. Mauna Loa Solar Observatory (MLSO) Mk4 coronameter $p B$ data displayed in polar coordinates, with solar latitude along the $x$-axis and altitude above the photosphere along the $y$-axis. The measurement strip for the cavity rim is shown in black, while the measurement strip for the cavity is shown in blue (dark gray in print edition). Note the curvature of the cavity rim measurement strip that excludes data from points outside of the cavity rim.

(A color version of this figure is available in the online journal.)

modeled as a torus that partially encircles the Sun at a constant solar latitude. Using this model, we can predict which locations along a chosen line of sight are occupied by cavity material and which locations are occupied by cavity rim material. We can then make the appropriate corrections and invert the $p B$ data to calculate the density of both the cavity and the cavity rim. Not every cavity fits the requirements of this model, and a cavity must be carefully chosen if it is to be compatible with these inversion techniques. See Fuller et al. (2008) for a more thorough description of the model geometry and the inversion process.

\section{MINOR MODIFICATIONS TO THE INVERSION PROCESS}

In order to create a more accurate and versatile process by which to invert $p B$ data into density profiles, we have made two key adjustments to the method used by Fuller et al. (2008). The first change is to allow non-radial measurement strips for cavity $\operatorname{rim} p B$ data from the Mk4 coronameter (see Figure 1 for example measurement strips). Since the cavity rim typically bends around the cavity to different latitudes at higher altitudes, a radial measurement strip frequently falls out of the cavity rim and into the surrounding corona at higher altitudes. We now select a curved measurement strip in order to obtain a more accurate sampling of cavity rim material throughout the full range of altitudes in the measurement. We can still use the model geometry to calculate the appropriate $p B$ correction for each pixel in the measurement strip. This technique allows us to measure the density of the cavity and cavity rim right up to the top of the cavity.

The second adjustment to the inversion process is to scale the correction factors that account for projection effects of the cavity rim to the brightness of the cavity rim. In the original method, the correction factors to the $p B$ measurements for cavity material were calculated using the density of a helmet streamer as calculated by Gibson et al. (1999). However, the density in a helmet streamer (and thus the cavity rim) differs from streamer to streamer, so the correction factor should also differ. In the new inversion process, for each system we measure the $p B$ of the cavity rim at $1.2 R_{\odot}$ and calculate its ratio with the $p B$ at the same height of the helmet streamer measured by Gibson et al. (1999). We then multiply the density of the streamer of Gibson et al. (1999) by this ratio in order to calculate correction factors to $p B$ measurements. We utilize the $p B$ at a height of $1.2 R_{\odot}$ because this altitude is contained in the measurement strips of every cavity analyzed in this article.

\section{CAVITIES USED IN ANALYSIS}

We have chosen 24 white-light cavity systems from MLSO Mk4 coronameter data on which to perform a density analysis in this article. The cavities were chosen primarily based upon the criteria outlined in Fuller et al. (2008). The most important criterion is that the cavity be visible for a sufficient number of days to satisfy (or nearly satisfy) the requirements for axisymmetry. Cavities failing to meet the strict axisymmetry requirements can still be analyzed, but will have larger uncertainties associated with the inversion process (see Fuller et al. 2008). It was also important that the cavities maintained roughly constant shape and latitude throughout these dates, and preferably the cavities existed relatively near the equator. While larger cavities exhibit smaller measurement errors and make for more accurate density calculations, we also included several smaller cavities in our analysis in an attempt to search for correlations between cavity size and other cavity features.

Table 1 displays a table of cavity properties. The cavities range in date from 2002 December (in the declining phase from solar maximum) to 2008 September (near solar minimum) in order to determine if cavity properties are related to the solar magnetic cycle. The tops of the cavities range in altitude from $0.248 R_{\odot}$ to $0.592 R_{\odot}$ above the photosphere. Furthermore, the cavities exhibit a wide range of latitudes and brightnesses and represent a diverse sampling of coronal cavities.

\section{CAVITY MORPHOLOGY}

The cavity morphology we have assumed thus far is that a cavity is shaped like a torus at constant latitude that partially encircles the Sun. This torus shape implies that the cavities will have a perfect circular cross section in the plane of the sky, and so they will appear as circles in coronagraph images. Furthermore, this torus is modeled to extend down to $1.0 R_{\odot}$ so that it is tangent with the solar photosphere. While cavities usually do maintain a roughly circular shape, there are inevitable deviations from the geometry of this model.

One way to analyze these deviations is to measure the size of the cavity as it appears in coronagraph images in terms of both cavity width and the altitude of the top of the cavity. According to our model, these measurements should produce equal values of cavity radius, but there are significant variations for real cavities. These deviations can be used to study cavity morphology, how it relates to cavity properties, and the mechanisms underlying cavity formation. To measure the altitude of the top of the cavity, we simply estimate the location of the top of the cavity in Mk4 $p B$ images like those shown in Figure 2. Although the top of the cavity is not evident in a radial $p B$ profile, the Mk4 $p B$ images shown in Figure 2 have a radial vignetting function applied, causing the top of the cavity to stand out and be measured. Because of the occulting disk we cannot see the cavities near the photosphere, so we do not know if the cavities extend all the way 


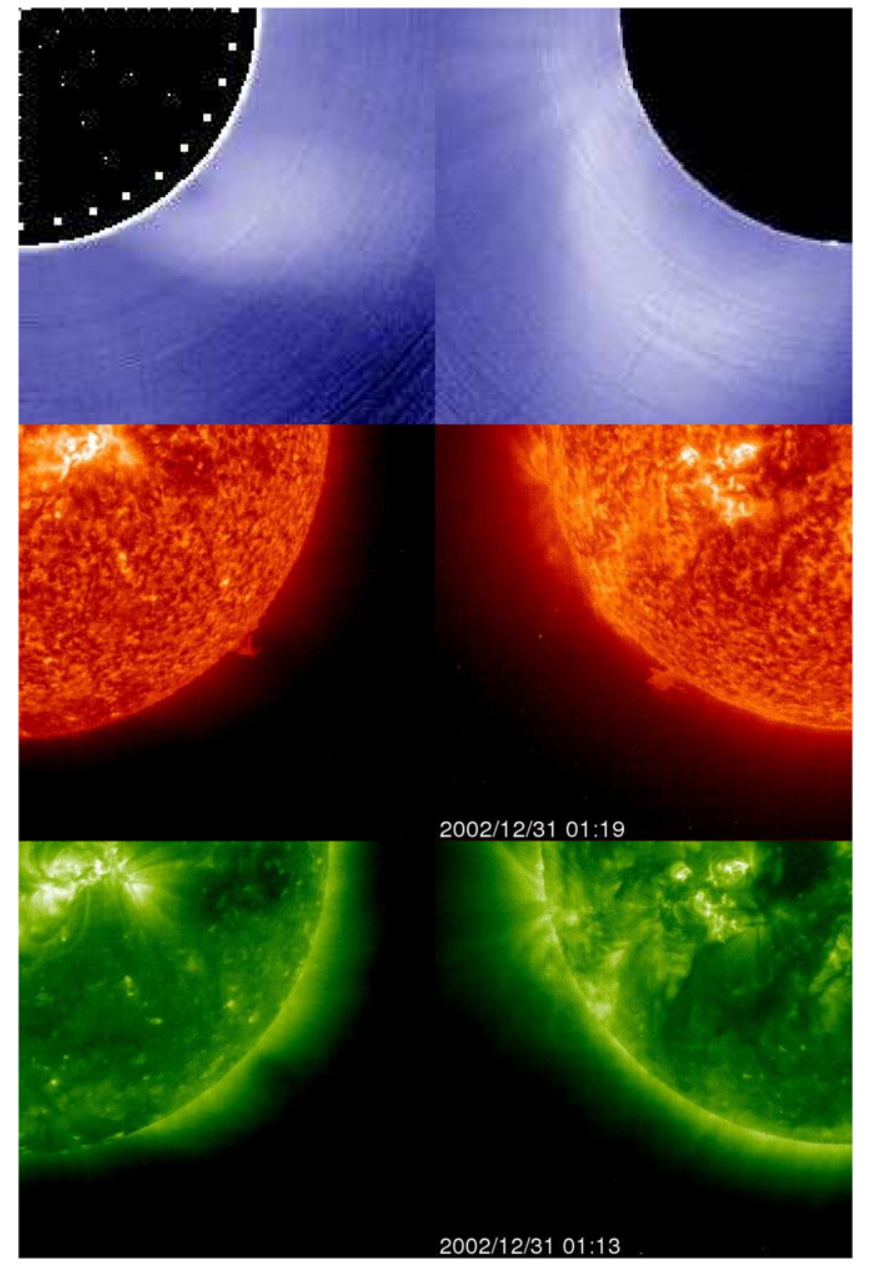

Figure 2. Small cavity (left) and a large cavity (right) imaged in white-light $p B$ from the MLSO Mk4 coronameter (top), and by the EIT telescope onboard the $\mathrm{SOHO}$ satellite, at $304 \AA$ (middle) and $195 \AA$ (bottom). The small cavity was measured on 2008 January 8, while the large cavity was measured on 2002 December 31. In the $304 \AA$ images, the underlying prominences of the cavities are clearly visible. The small cavity is visible at $195 \AA$ but the large cavity is not.

(A color version of this figure is available in the online journal.)

down to the photosphere in white light. However, many cavities are visible at low altitudes in EUV wavelengths (see Figure 2), and it appears that cavities' bottoms do lie near the photosphere in the cases we have examined. Consequently, we will refer to the altitude of the top of the cavity as the cavity height. To determine cavity width, we use the Mk4 images to measure the angular extent of the cavity at its widest point (which for most of our cavities is at or near $1.2 R_{\odot}$ ). The cavity width is then the length of the chord spanning the angular width of the cavity at the altitude where the angular width measurement is performed.

Figure 3 shows a plot of cavity height versus cavity width for the 24 cavities. The black line is made up of points where cavity height is equal to cavity width, so that if the cavities were perfectly circular and tangent to the photosphere they would lie along this line. It is clear that cavities with smaller widths lie to the left of the line, while cavities with larger widths generally lie to the right of the line. So, assuming a cross section tangent to the photosphere, narrow cavities are usually thinner than a circle while very wide cavities are fatter than a circle. Correspondingly, we can conclude that cavities with a small height are thinner than a circle, but we cannot say definitively whether tall cavities are thinner or wider than a circle. These

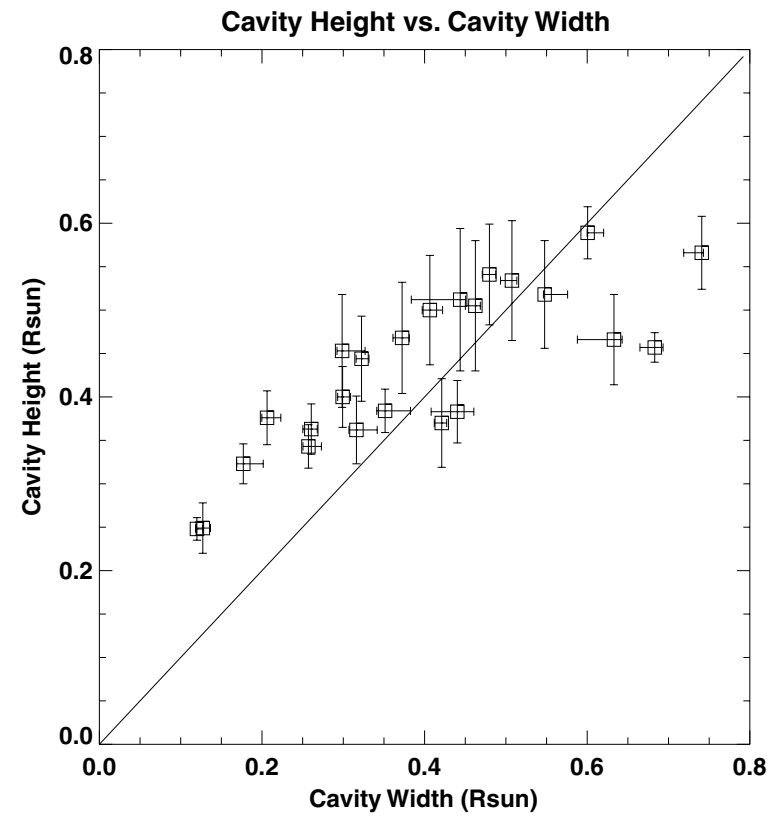

Figure 3. Cavity height vs. cavity width for our sample. The diagonal line denotes points where cavity width and cavity height are equal.

results are consistent with those of Gibson et al. (2006). While cavity height steadily increases with cavity width for smaller cavities, it appears that cavities do not have heights in excess of about $0.6 R_{\odot}$ even though their widths have been measured up to about $0.8 R_{\odot}$. This trend in cavity morphology may have important implications for cavity stabilization. At the short end of the spectrum, cavity width increases nearly proportionally to cavity height until a height of about $0.5 R_{\odot}$ is reached. At this point, cavities rarely become taller, although they can continue to become wider.

It is possible that the widest cavities only appear wide because they are not centered upon a constant latitude, i.e., their axis is not parallel to the solar equator as we have assumed in our model. Such cavities would appear wider than a circle because they would subtend a wider range of latitudes throughout their longitudinal extent, but they would never appear to extend beyond the altitude of the cavity top. If tall and wide cavities are observed to vary in latitude on different days of observation, it is possible that their wideness is due primarily to this effect. However, short cavities with significant latitudinal variation would become obscured by the cavity rim, and so in accordance with observations, we would not see any short and wide cavities. To test this theory, we have calculated the standard deviation in central colatitude for days surrounding the measurement date of the five widest and five thinnest cavities in the sample. The standard deviation for the wide cavities is 2.34 , while for the narrow cavities it is 2.07 . The widest cavities do not change latitude significantly more than the small cavities, and the small increase in standard deviation is to be expected because it is more difficult to measure the central latitude of very wide cavities. Thus, while it is possible that some very wide cavities appear widened due to latitudinal variation, it appears that some cavities naturally form with larger widths than heights.

It is also interesting to note how cavity width depends on the date at which the cavity existed. Figure 4 displays a plot of cavity width as a function of cavity date. At earlier dates during the decline from solar maximum, cavities occupy the full range in cavity width, while cavities at later dates near 


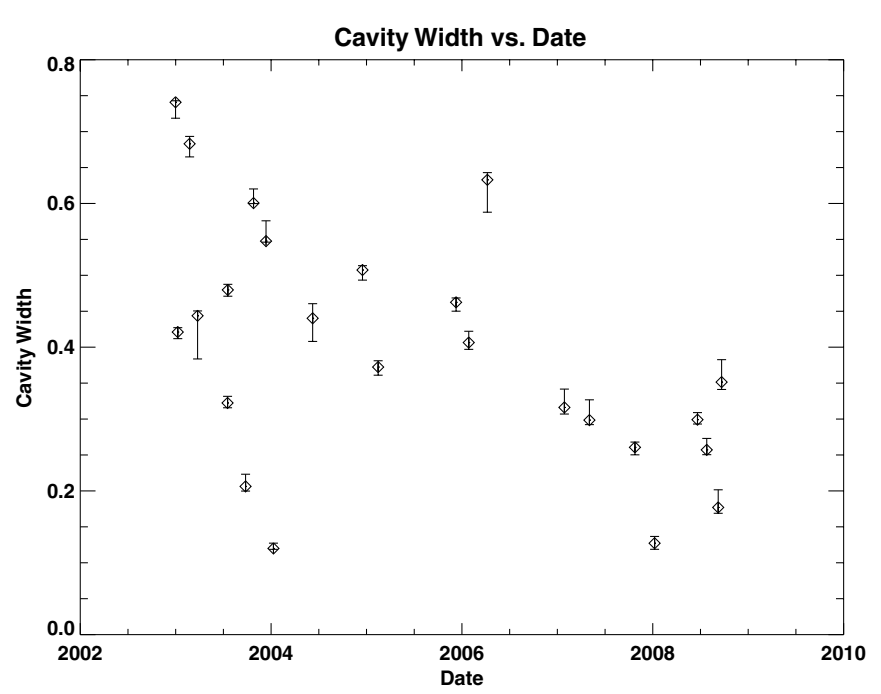

Figure 4. Cavity width as a function of cavity date. Cavities of all shapes and sizes are produced near solar maximum, while smaller and thinner cavities are more common near solar minimum.

solar minimum tend to be shorter and skinnier. The cavities we observe near solar minimum also are observed to lie at similar latitudes (around $45^{\circ}$ from the poles). During solar maximum, the Sun's corona is much more complex and the prominences associated with our cavities are more varied in size, shape, and latitude. It is not surprising that the cavities are also more varied.

Our inversion technique assumes that the cavities have a circular cross section in the plane of the sky, with the bottom of the circle tangent to the solar photosphere. Therefore, the noncircular shape of the cavities as discussed above can affect our density calculation. To account for this non-circularity, we can model cavities as having an elliptical cross section in the plane of the sky. For thin (and therefore short) cavities, this model is justified because the cavities can be seen to extend down to the photosphere in EUV images, giving them a roughly elliptical shape (see Figure 2). Although wide (and therefore tall) cavities do not show up in EUV images, a much larger proportion of these cavities can be seen in Mk4 images, and the cavities appear roughly elliptical in these images. Since the line of sight never passes through altitudes lower than the altitude of the occulting disk, the shape of the cavities below the disk is unimportant for our density calculations. As long as the cavity appears elliptical at all altitudes above the lowest altitude measured (usually about $1.16 R_{\odot}$ ), the elliptical geometry is justified. Because small cavities are thinner than they are tall, we model them as having an elliptical cross section with semimajor axis in the radial direction. Conversely, cavities that have a larger width than height are modeled as having an elliptical cross section with semi major axis perpendicular to the radial direction. In Section 5.5, we present modified density calculations using the elliptical geometry and analyze the effect of the new geometry on cavity properties.

\section{RESULTS}

\subsection{Density Profiles}

The results of the density calculations reveal that the cavities exhibit significant diversity in their densities, just as they do in size, brightness, etc. The cavities have different densities at any chosen altitude, as do the cavity rims. It is useful to analyze the density depletion of each cavity with respect to the cavity rim, which is defined as

$$
\frac{\Delta N}{N}=\frac{N_{\text {rim }}-N_{\text {cav }}}{N_{\text {rim }}} .
$$

Although density depletions vary from cavity to cavity, these depletions do not exhibit as much diversity as the absolute densities of the cavities, and this is discussed below. See Figure 5 for three example density profiles. See Figure 7 for the density depletion profiles of these cavities.

There are some traits that are common to nearly all cavities. First, every cavity analyzed in this article has a flatter density profile than does its cavity rim, so the density of the cavity falls off slower with height than does the density of the cavity rim, yielding a negative slope for the depletion profile. In other words, the depletion is largest at the lowest altitude measured (usually around $1.16 R_{\odot}$ ), and the depletion is smallest at the highest altitude measured (which varies according to cavity size from about $1.25 R_{\odot}$ to nearly $1.6 R_{\odot}$ ). Furthermore, for every cavity except the smallest two, the depletion approaches zero near the top of the cavity, i.e., the cavity and cavity rim have the same density at the top of the cavity (Figure 8). This implies that there is a jump in density from cavity to cavity rim at the top of the smallest two cavities, whereas the densities of cavity and cavity rim merge smoothly at the tops of most cavities.

Another important result is that, as was found for the single case analyzed in Fuller et al. (2008), the density of both cavity and cavity rim is much larger than that of a coronal hole for all 24 cases studied here, and usually larger than the density of the helmet streamer analyzed by Gibson et al. (1999). The cavity and cavity rim do not appear much denser than the helmet streamer density shown in red in the above plots because this density has been scaled by the process outlined above to a new, usually higher density. The mean cavity density at $1.2 R_{\odot}$ is 4.85 times greater than the coronal hole density at the same height as measured by Guhathakurta et al. (1999). The fact that the cavity density is much larger than that of a coronal hole shows that cavities have quite high densities despite their darkened appearance. They only appear dark in comparison to the bright cavity rims around them, which almost always have densities equal to or greater than that of the streamer of Gibson et al. (1999). Since the density of the helmet streamer of Gibson et al. (1999) is almost always less than the densities of the cavity rims, it seems likely that the helmet streamer of Gibson et al. (1999) had an unusually low density. The fact that the helmet streamer of Gibson et al. (1999) occurred during solar minimum may be correlated with its low density (see next section).

\subsection{Cavity Date}

One of the purposes of examining cavities during different phases of the solar magnetic cycle is to determine how cavity properties vary or remain constant during differing solar magnetic environments. The dependence of cavity properties on their date of occurrence may therefore shed light onto how cavity formation is related to the solar magnetic field.

Let us start by examining the densities of cavity systems at different dates. Figure 6 plots the density for both cavity (blue) and cavity rim (orange) at $1.2 R_{\odot}$. Once again, the height of $1.2 R_{\odot}$ is contained in the density analysis for all cavities, so we use it as a common reference point at which to compare cavity properties. We find that cavity systems existing earlier (and hence closer to solar maximum in our study) tend to have slightly larger values of density at $1.2 R_{\odot}$. However, this trend 

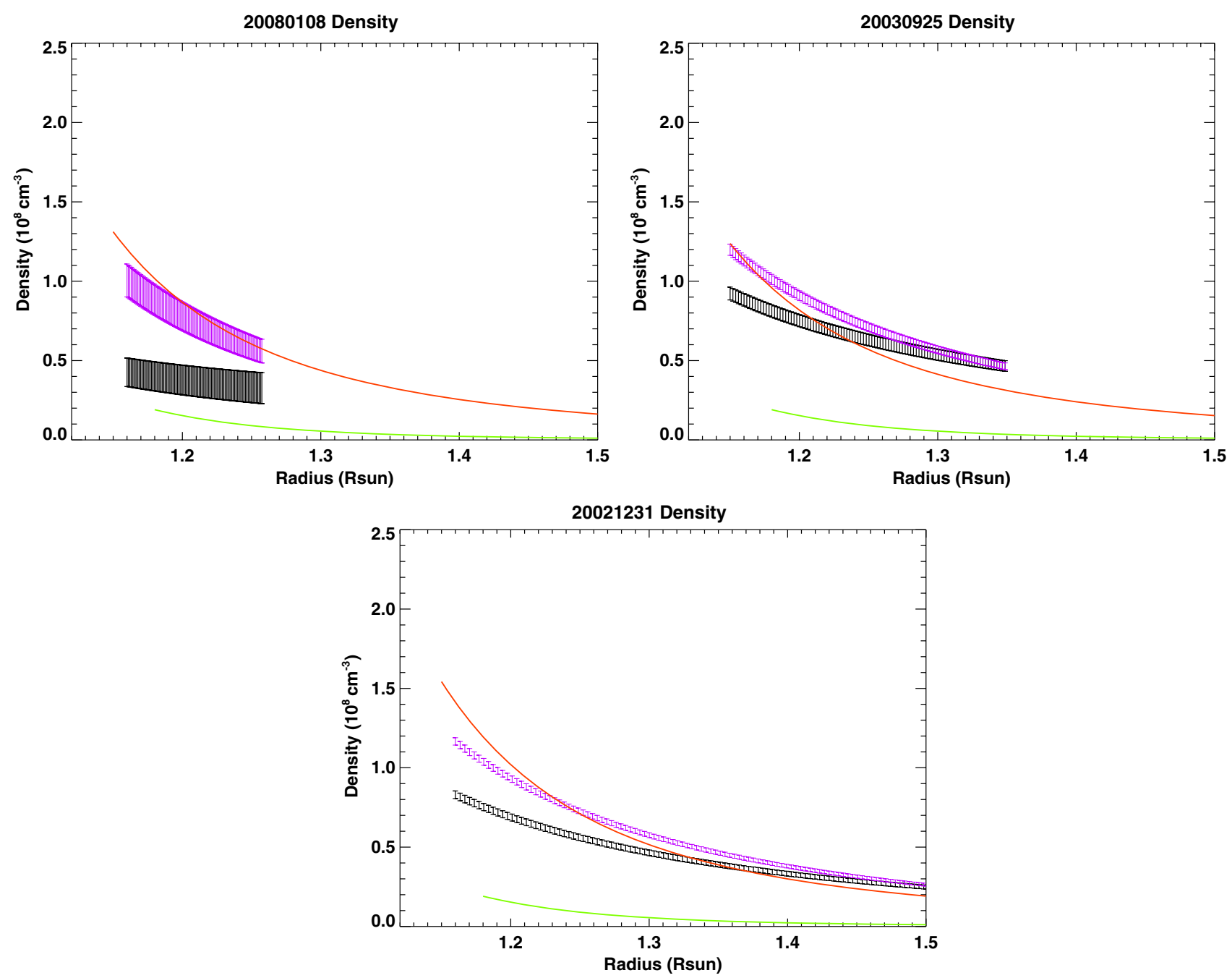

Figure 5. Density profiles for the cavity (black/dark) and cavity rim (purple/light) for the cavity systems observed on 2008 January 8 (top), 2003 September 25 (middle), and 2002 December 31 (bottom). The green/thin line is the density of a coronal hole as calculated by Guhathakurta et al. (1999), while the red/thick line is the scaled density of the helmet streamer of Gibson et al. (1999).

(A color version of this figure is available in the online journal.)

is not especially pronounced, and the more noticeable feature is that cavities near solar maximum span a wider range in density. So, while the increased solar magnetic activity is capable of producing denser systems, the main effect is that it produces more diverse cavity systems with a higher variance in density. Figure 6 also displays the density depletions of the cavities relative to their cavity rims. Despite the variance in density, the cavity systems do not exhibit any remarkable trends in density depletion at $1.2 R_{\odot}$.

Figure 6 also displays scale height temperatures of both cavity and cavity rim, calculated according to the method outlined in Fuller et al. (2008). Scale height temperatures only accurately represent the local plasma temperature if it is in hydrostatic equilibrium, and if temperature is constant along magnetic field lines, which may not be good assumptions. They are related to the slope of density versus height (flatter density profiles have higher scale height temperatures). We provide these temperatures for reference purposes only, as discussed in Fuller et al. (2008), white-light data is insufficient on its own to constrain temperature, and the flat cavity density profile could also arise in an isothermal system (where cavity and cavity rim have the same temperature). The example was given in that paper of an axisymmetric magnetic flux rope, in which a radial density profile within the cavity crossed concentric field lines of increasing relative boundary density, leading to a flatter density falloff than in the surrounding rim. The temperatures calculated in this manner follow a similar pattern to the density, with the earlier systems having slightly larger temperatures. Note that the scale height temperature of the cavity is larger than that of the rim, so the temperature depletion of cavity relative to rim is negative. Similar to the $p B$ and density depletion, the temperature depletion lies between the values of 0 and -0.4 with no obvious trends, except for the two obvious outlying systems that are discussed in Section 5.4. Studies of cavities seen in EUV or soft X-ray might prove more useful to probe the properties of these smaller cavities because they do not have an occulting disk obscuring lower altitudes as do white-light observations.

\subsection{Cavity Size}

As mentioned above, cavity size is an important tool for examining cavity formation, so it would be helpful to know which cavity properties change with cavity size and which properties are common to all cavities. To start, let us examine the density depletion profiles of a small, medium, and large cavity 

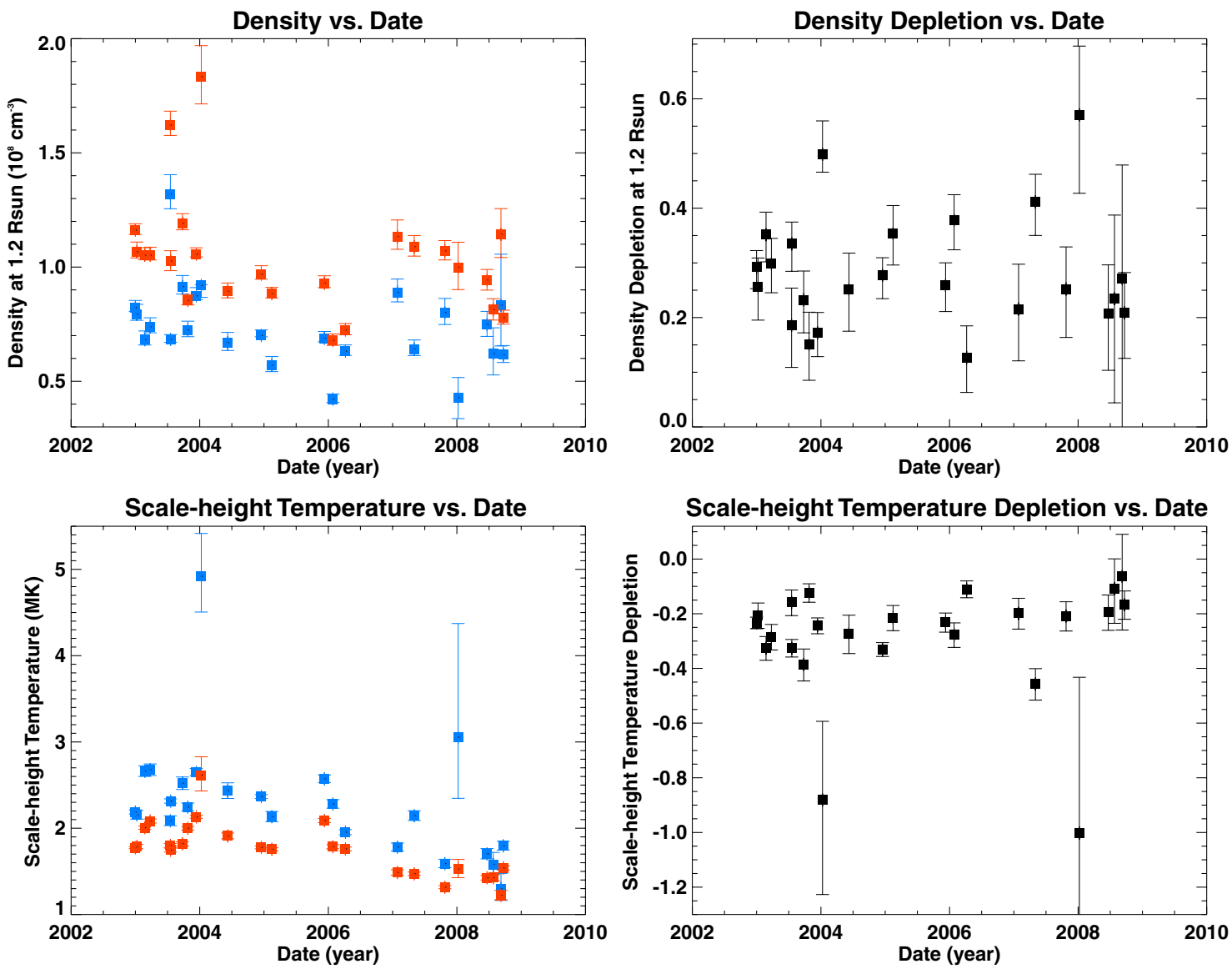

Figure 6. Top left: density of the cavity (blue) and rim (orange) at $1.2 R_{\odot}$ as a function of date for all 24 cavity systems. Top right: corresponding density depletion of cavity relative to the rim, where density depletion is given by $\frac{\Delta N}{N}=\frac{N_{\text {rim }}-N_{\text {cav }}}{N_{\text {rim }}}$. Bottom left: scale height temperature of cavity (blue) and rim (orange). Bottom right: corresponding scale height temperature depletion of cavity relative to the rim.

as shown in Figure 7. First of all, notice that the depletion of the larger cavities can be measured to much larger heights because the systems extend to higher altitudes, but that measurements for all cavities have a lower limit of around $1.16 R_{\odot}$ due to the occulting disk of the Mk4 coronameter. Consequently, we have much less data for smaller cavities, and so the error bars are much larger (as discussed above). When looking at the depletion profiles, it is immediately obvious that the smallest cavity has much larger depletion values, while the two larger cavities are closer, with overlapping error bars.

Another distinguishing feature that naturally arises from cavity size is the slope of the density depletion profile. From the top plot of Figure 7 it is clear that the smallest cavity has the steepest slope, while the largest cavity has the flattest slope. Since most cavities have similar density depletions at $1.2 R_{\odot}$ and most cavities have almost zero density depletion at their tops, most cavities have a similar change in depletion from $1.2 R_{\odot}$ to their tops. However, this change occurs over a smaller altitude range for shorter cavities, and so these cavities have a steeper slope to their density depletion profiles.

To further understand this idea, we have also plotted the density depletion profiles for the same three cavities with the altitude range normalized to each cavity's height, so that a value of 1.0 corresponds the top of each cavity. It is evident that we can only measure the top parts of small cavities, while for large cavities we can measure a much larger proportion of the cavity material. The normalization produces a remarkably similar slope for the depletion profiles of all three cavities but does not change the markedly greater density depletion of the small cavity, and this feature will be discussed below.

To find more trends in cavity size, we have plotted the central depletion, the minimum depletion, the mean depletion, and the normalized depletion slope for each cavity as a function of cavity height in Figure 8. The central depletion is the depletion at one half the cavity height above the photosphere, while the minimum depletion is the depletion at the top of the cavity. For the smallest two cavities, the central altitude (located at $1.124 R_{\odot}$ for both small cavities) is below the occulting disk, so we have used the depletion at the lowest altitude measured $\left(1.14 R_{\odot}\right.$ for the cavity measured on 2004 January 10 and $1.16 R_{\odot}$ for the cavity measured on 2008 January 8). The normalized depletion slope is calculated by multiplying the depletion slope by the height of the cavity. If the depletion profile is linear at all heights, the normalized depletion slope represents an estimate of the change in depletion from the photosphere to the top of the cavity.

With the exception of the smallest two cavities, the central depletion of the cavities lies between 0.09 and 0.32 , with no apparent relation to cavity height. If the centers of the smallest two cavities were within the measurement ranges, we would likely measure an even greater central depletion for these two cavities since we would be measuring the depletion at a lower altitude. As noted above, nearly all the cavities (except the smallest two) have a minimum density depletion near zero. While a minimum depletion of zero is within the error bars of most of the cavities, the mean depletion at the top of the cavities is $\frac{\Delta N}{N}=0.077$, so the average cavity has only a very 

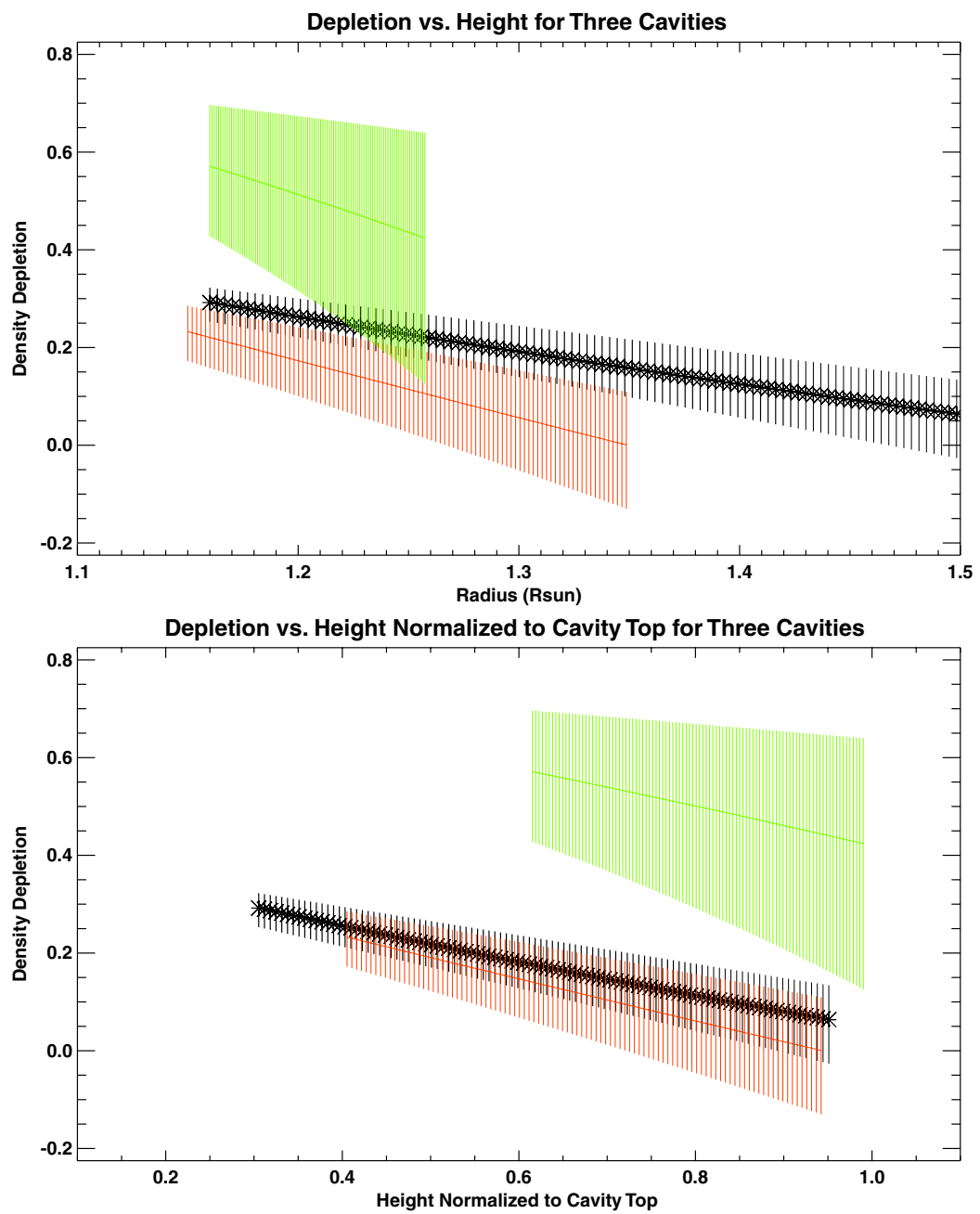

Figure 7. Top: density depletion profile for the cavities measured on 2008 January 8 (green/darkest), 2003 September 25 (orange/dark), and 2003 December 12 (black/lightest). Bottom: density depletion profiles of the same cavities normalized to cavity height.

(A color version of this figure is available in the online journal.)

small density jump from cavity to cavity rim at the top of the cavity. The mean depletion of all the cavities (except the smallest two) lies between 0.07 and 0.29 , again with no apparent relation to cavity height. The normalized depletion slope shows little dependency on cavity height, but in this case the smallest two cavities are not outliers, as discussed above. In general, cavity depletion does not appear to be strongly correlated with cavity size (except for the smallest two), and cavity depletion is neither tightly constrained (depletions vary by a factor of roughly 3 ), nor does it exhibit a large range of variation (depletions only vary by a factor of 3). It should be noted that the density depletion error bars are nearly as large as the variations in depletion between cavities, so we cannot tightly constrain variations in density depletion between cavities. Viewed in this light, the cavities (excluding the smallest two) have very similar density depletions within error bars.

\subsection{Small Outlying Cavities}

As mentioned above, the two smallest cavities have exceptional properties compared to the other cavities in the sample, including a very high central, minimum, and mean depletion, as well as a very negative depletion slope. These properties appear to exist despite the fact that the uncertainties are very large for these cavities. We must therefore ask whether these differences are a natural feature of very small cavities, or whether the two smallest cavities in this sample are simply abnormal.

It is possible that the large depletions of the smallest cavities are a result of selection effects when looking for cavities to analyze. Smaller cavities are harder to see, so they must be more depleted to stand out in a coronameter image. Thus, only highly depleted small cavities had a high enough contrast to be well resolved in coronameter images and be selected for our density analysis. However, it is also possible that small cavities are naturally more depleted than large cavities due to magnetic or temperature effects of cavity formation. For instance, a small cavity may be associated with a strong magnetic field, producing a high magnetic pressure within the cavity balanced by the large density depletion (Low \& Berger 2003).

Gibson et al. (2006) also found that small cavities have larger $p B$ depletions and a seemingly different structure than large cavities. Furthermore, the two small cavities have radial $p B$ profiles that differ from the other cavities and indicate a jump in density at the top of the cavity. Whereas in most cavities the $p B$ falls off smoothly with height so that the cavity top is not evident in the measured $p B$ profile (Figure 9), there is a noticeable flattening or small peak in the $p B$ at the top of the small cavities. This $p B$ structure may imply a jump in density that appears evident in the density profiles (Figure 5). To reach a conclusion, we need more studies on small cavity systems 

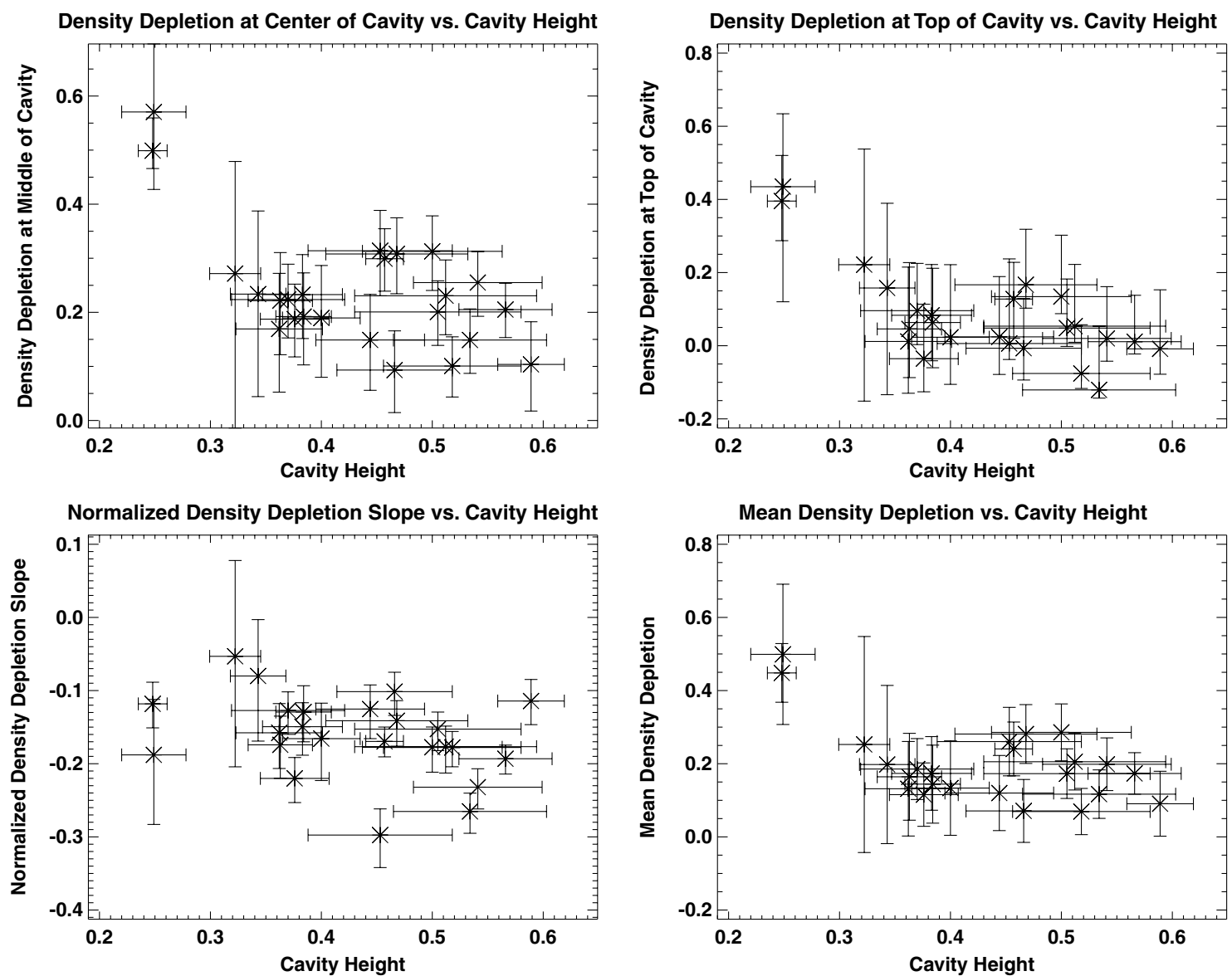

Figure 8. From top left: density depletion at the center of each cavity, density depletion at the top of each cavity, normalized density depletion slope, and mean density depletion of each cavity. The two smallest cavities appear to have quite different density properties, except in the plot of normalized density depletion.

(which are relatively hard to find) because we only analyze two very small cavities in this article.

\subsection{Modifications for Elliptical Cavities}

Thus far, the geometry that we have utilized in our $p B$ inversions assumes that the cavities have circular cross sections. However, cavities that have elliptical cross sections will deviate from this geometry because a given line of sight will intersect the edge of the cavity at a different scattering angle. We will continue to use circular geometry for the cavity rim. For an elliptical cavity of height $2 h$ and width $2 w$ that grazes the photosphere, the scattering angle corresponding to the edge of the cavity along a line of sight through the center of the cavity is given by

$$
\alpha_{c}= \pm \arctan \sqrt{\frac{h^{2} w^{2}+2 h w x(1+h) \sin \theta_{c}}{x^{2}(1+h)^{2}}}
$$

where $x=\sqrt{h^{2} \sin ^{2} \theta_{c}+w^{2} \cos ^{2} \theta_{c}}$ and $\theta_{c}$ is the colongitude of the center of the cavity. This equation assumes that the cavity is close enough to the equator that the line of sight only intersects the cavity at two points (see Fuller et al. (2008) for more discussion of cavity geometry).

Using the above equation, we see that a cavity that is wider than a circle will have a higher scattering angle at the location where the line of sight intersects the edge of the cavity than it would if we used circular geometry with $R_{\text {cav }}=h$. Consequently, the cavity rim projects into the line of sight at a higher scattering angle than it does in our original calculations. Therefore, the corrections we added to $p B$ measurements were too large, and the cavity will be slightly denser than we originally calculated. However, since our original $p B$ corrections were too large, our original error bars were overly generous, and the real value of the cavity density will lie within our original uncertainties.

For cavities that are thinner than a circle, the line of sight intersects the edge of the cavity at a smaller scattering angle than originally calculated using $R_{\mathrm{cav}}=h$. This means that the cavity rim projects into the line of sight at smaller scattering angles, and so our original $p B$ corrections were too small. So, thin cavities will be less dense than originally calculated and their densities may lie outside the range of our uncertainties.

To quantify the change in density resulting from elliptical cavity geometry, we calculate the ratio $\alpha_{\text {ellip }} / \alpha_{\text {circ }}$, where for a line of sight through the center of the cavity $\alpha_{\text {ellip }}$ and $\alpha_{\text {circ }}$ are the scattering angles at the edge of the cavity using elliptical geometry and circular geometry, respectively. Then, for each line of sight (each pixel in the measurement strip), we multiply the scattering angle calculated according to circular geometry by this ratio. Thin cavities will thus have smaller scattering angles (and larger $p B$ corrections) than originally calculated, while larger cavities will have larger scattering angles (and smaller $p B$ corrections). We multiply by the ratio $\alpha_{\text {ellip }} / \alpha_{\text {circ }}$ for all lines of sight because calculating the exact scattering angle for lines of sight not through the cavity center is more difficult with elliptical geometry.

The results of the new density inversions are displayed in Figure 10 for a very thin cavity and a very wide cavity. As 

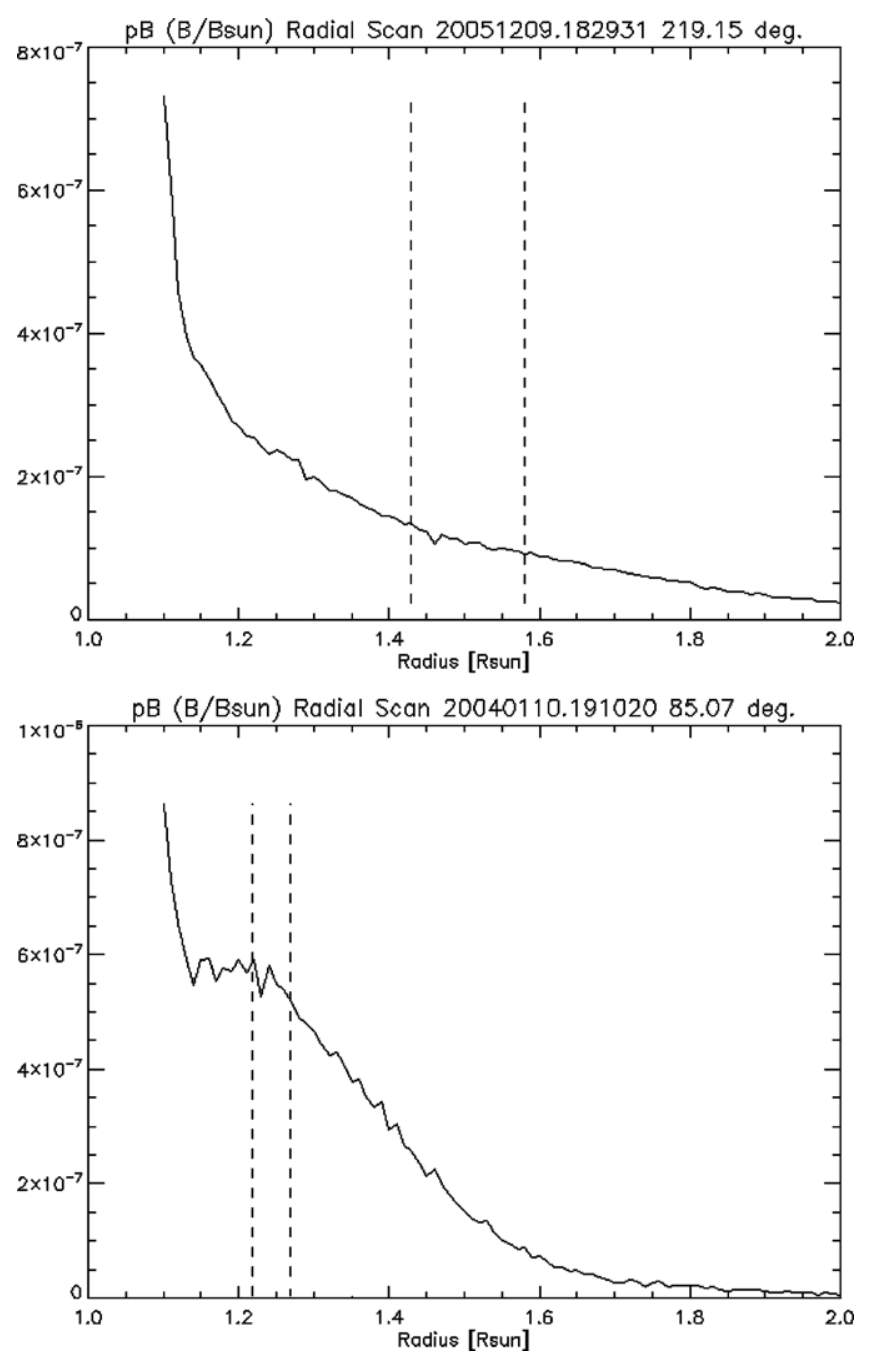

Figure 9. Top: $p B$ scan as a function of radius through the center of the tall cavity measured on 2005 December 9 . The dotted line indicates the location of the top of the cavity. Bottom: $p B$ scan as a function of radius through the center of the short cavity measured on 2004 January 10 . Note the evident flattening, or perhaps slight peak in $p B$ that makes the location of the cavity top evident.

predicted, the density of the thin cavity became lower, while the density of the wide cavity became higher. However, in both cases the uncertainties of the new calculations overlap with the uncertainties of the original calculations. Also notice that the density of the rim became slightly larger for the thin cavity and slightly smaller for the wide cavity. Since these two cavities are the most narrow and most wide of the cavities in our sample, we expect the deviations from our original calculations to be largest for these two cavities. Since the uncertainties of the original and new density calculations overlap, our original calculations are not greatly affected.

It is interesting to speculate how elliptical geometry may change the observed trends (or lack thereof) of cavity density as a function of cavity size. The effect of elliptical geometry is to make thinner cavities more depleted at all heights and create a steeper depletion slope. Conversely, wider cavities will become less depleted at all heights with a flatter depletion slope. If the corrections were applied to all the cavities in the sample, it is possible that we would see a trend where the smaller cavities have higher mean, top, and bottom depletions, as well as more negative depletion slopes. In this case, the two small outliers may not be exceptional in their properties, but rather represent
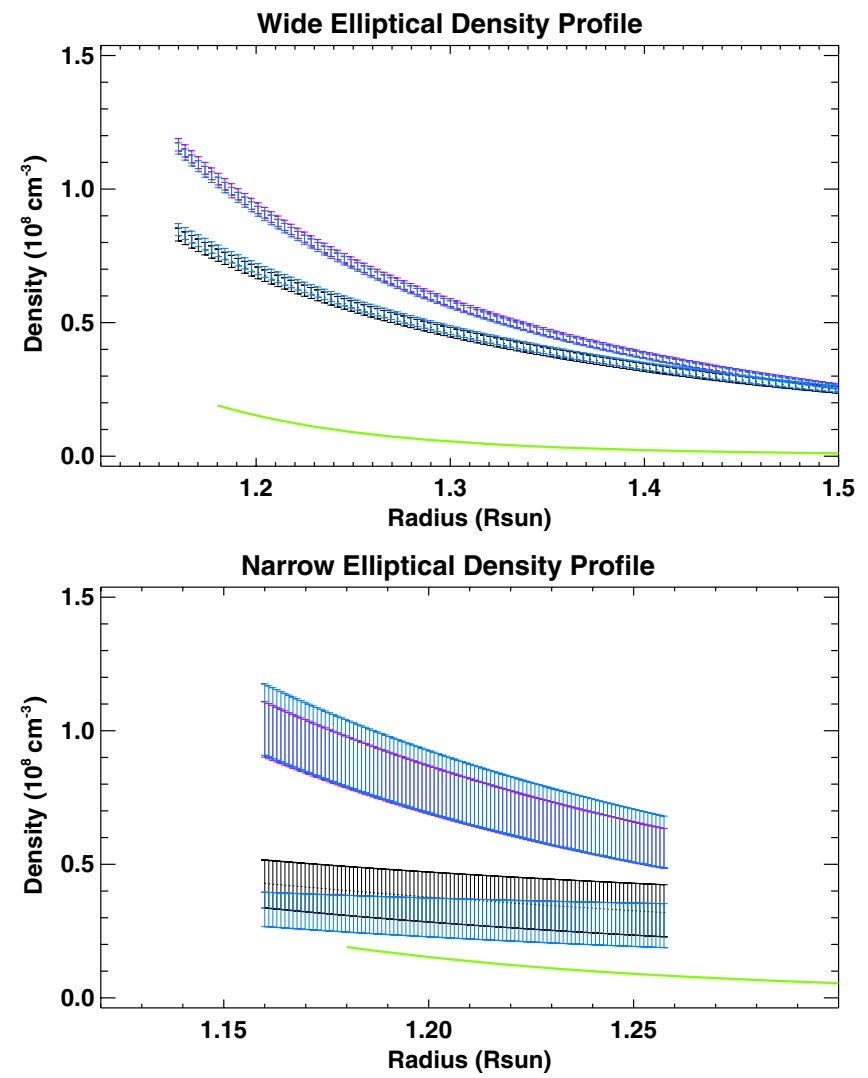

Figure 10. Top: density profile for the wide cavity (black) of 2002 December 31 , and cavity rim (purple) overlaid with new density profiles utilizing corrections for an elliptical cross section (blue). Bottom: density profile for the narrow cavity of 2008 January 8 , with the same color scheme.

an extreme example of this trend. To verify this idea, more accurate elliptical corrections to cavity geometry are needed. In particular, a numerical cavity model could be used to accurately calculate the scattering angle at the edge of the cavity and perform the appropriate $p B$ inversions.

\section{CAVITY PRESSURE}

Since quiescent cavities are stable coronal structures that can maintain their shape and size for days or weeks at a time, there cannot exist a significant total pressure difference between cavity and cavity rim, where total pressure is defined as the sum of thermal and magnetic pressures. In order to accurately calculate the pressure of the cavity and the cavity rim, we would need to have density, temperature, and magnetic field strength data. Until such data become available, we content ourselves with testing the potential validity of possible models, for example that of hydrostatic balance as discussed in Fuller et al. (2008), where cavity temperature is calculated from radial density profiles.

Figure 11 shows the pressure profile for a typical cavity and cavity rim with the temperature determined in the manner described above. Unlike the density profile, there is very little difference between cavity and cavity rim throughout the entire height range. In fact, for most of our cavities the pressures of cavity and cavity rim are equal within error bars at nearly all heights because the higher scale height temperature predicted for such a model compensates for the density depletion, resulting in continuous thermal pressure. Some cavities do exhibit slight deviations from continuous thermal pressure, as the average 


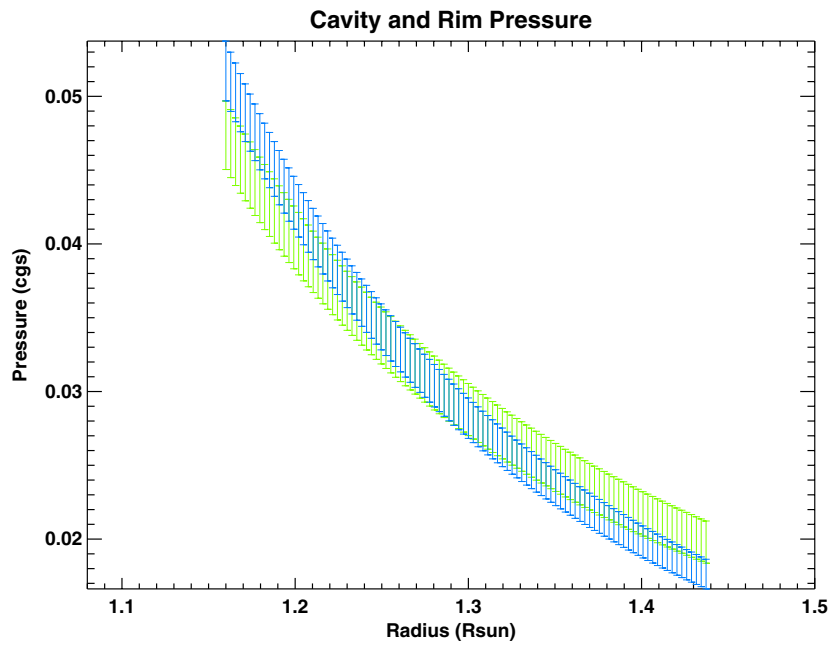

Figure 11. Cavity pressure (green) and cavity rim pressure (blue) as a function of height for the cavity observed on 2005 December 9.

pressure depletion is $0.098 \pm_{0.188}^{0.121}$ at the bottom of the cavity, meaning the cavities have slightly lower pressure than the cavity rims at the lowest height measured. At the top of the cavities, the average pressure depletion is $-0.173 \pm_{0.240}^{0.267}$, meaning the cavities have slightly higher pressure than the cavity rims at the greatest height measured. However, in both cases a mean pressure depletion of zero is within the error bars.

\section{CONCLUSIONS}

We have extended a prior analysis (Fuller et al. 2008) to calculate density profiles of 24 cavity systems observed in white light, thus demonstrating the robustness and generality of these previous results which were obtained for a single cavity system. We have implemented minor modifications to the method of Fuller et al. (2008) as described in Section 2, in order to enable its application to a broad variety of cavities. For all cases studied, cavities were more than twice as dense as coronal holes at $1.2 R_{\odot}$ (4.85 times more dense on average), and possessed a mean depletion relative to their surrounding streamers of $28 \%$ at this altitude. Also for all cases studied, cavity density fell off with a flatter radial profile than that of the surrounding streamer in a manner consistent with hydrostatic models where cavities are hotter than their surrounding streamer by an average of $28 \%$. Thus it is possible that high temperatures within cavities play a significant role in maintaining pressure continuity across their boundaries. However, as discussed in Fuller et al. (2008), white-light observations are insufficient on their own to establish cavity temperature, and independent temperature (or magnetic field) diagnostics are required to truly probe the relative roles played by gas versus magnetic pressure in cavities.

The majority of cavities possess similar density depletions at all heights, and also, as in the case studied by Fuller et al. (2008), have essentially zero depletion (cavity versus rim) at their tops. This is an important constraint on magnetohydrodynamics (MHD) and thermodynamic models of coronal cavities. However, there were two exceptions. These were the smallest cavities we observed, with a clear gap between their cavity heights and the heights of the rest of the cavities. We have discussed how selection effects might lead to the smallest cavities necessarily being the most depleted and thus most visible of the ones we have surveyed, but regardless of whether there may be small cavities that are less depleted that we did not find, it is a fact that we did not find any large cavity possessing such large depletions as these two small ones. It is possible that this relates to the relative strengths of magnetic fields in smaller versus larger scale coronal structures, and/or a dependency of thermal effects (relative heating/cooling terms) on cavity size. It would be worthwhile to pursue the connection between size and depletion further using soft X-ray (SXR) or EUV observations of cavities, which are not limited by the coronagraph occulter as white-light observations generally are.

Cavity properties appear to have a slight dependence on solar cycle, with the dimmest, least dense, and coolest systems occurring near solar minimum. Cavity systems occurring during the descent from solar maximum are slightly brighter, denser, and hotter on average, but these systems have a much greater variance in their properties. Thus, larger and brighter cavity/ rims occur near maximum, in addition to those of the smaller size/brightnesses that are seen throughout the cycle.

We have also found that wider cavities tend to extend to larger heights, while narrow cavities are restricted to lower heights. However, the range of observed cavity angular widths is between 0.12 and $0.76 R_{\odot}$, while cavity heights only vary between 0.24 and $0.6 R_{\odot}$. This implies that cavities do not in general fit the model of possessing circular cross sections whose bottoms graze the solar surface. Utilizing a model in which cavities possess an elliptical cross section whose bottom grazes the solar surface, we have calculated approximate corrections to $p B$ measurements and used these corrections to create new density profiles for a very narrow and a very wide cavity. The corrected density profile for the wide cavity hardly varies from our original density profile, while the density profile for the narrow cavity shows more variation but remains within experimental uncertainties of our original density profile. We suggest using a numerical model for the cavity to determine more accurate correction factors for cavities with non-circular geometry.

Finally, we have utilized the method outlined in Fuller et al. (2008) to calculate thermal pressure profiles for the cavity and cavity rim in all 24 cavity systems. The thermal pressure of cavity and cavity rim are equal within uncertainties at all heights for most of the cavities in our sample, using scale height temperatures determined from white light. The implication is that, if cavities tend to be hotter than their denser surroundings, thermal pressure and thus also magnetic pressure may be continuous across the sharply defined cavity boundary. The sharpness of the boundary may still reflect a magnetic flux surface, at which for example where there is a discontinuity in field line length (e.g., a separatrix surface, see Low \& Wolfson 1988 and Titov \& Demoulin 1999). Sharp changes in field line length may lead to thermal gradients from cavity to rim, see e.g., van Ballegooijen \& Cranmer (2008). To fully resolve these interesting questions of the interplay of cavity MHD and thermodynamics, independent methods of measuring cavity temperature and/or cavity magnetic field strength are required.

We thank Giuliana de Toma for internal HAO review of this manuscript. The Mauna Loa Solar Observatory is a facility of the National Center for Atmospheric Research and is sponsored by the National Science Foundation. $\mathrm{SOHO}$ is an international collaborative program of ESA and NASA. This work has benefited from discussions within the Coronal Prominence Cavity International Team (Leader, Sarah Gibson) of the International Space Science Institute (ISSI), Bern, Switzerland. 


\section{APPENDIX \\ CAVITY PROPERTIES}

Table 1

Cavity Properties Listed in Order of Date on which Density Inversions were Performed

\begin{tabular}{|c|c|c|c|c|}
\hline Measurement Date & Polar Angle & Height & Width & Before/After \\
\hline 2002 Dec 31 & $146.1 \pm 0.55$ & $0.566 \pm 0.042$ & $0.741 \pm_{0.022}^{0.002}$ & $2 / 3$ \\
\hline 2003 Jan 09 & $214.2 \pm 0.66$ & $0.370 \pm 0.051$ & $0.421 \pm_{0.009}^{0.006}$ & $3 / 4$ \\
\hline 2003 Feb 24 & $146.4 \pm 1.92$ & $0.457 \pm 0.017$ & $0.683 \pm_{0.018}^{0.010}$ & $1 / 2$ \\
\hline 2003 Mar 24 & $143.9 \pm 2.36$ & $0.512 \pm 0.082$ & $0.444 \pm_{0.060}^{0.007}$ & $3 / 2$ \\
\hline 2003 Jul 17 & $225.0 \pm 0.37$ & $0.444 \pm 0.049$ & $0.322 \pm_{0.007}^{0.009}$ & $2 / 2$ \\
\hline 2003 Jul 18 & $35.8 \pm 0.49$ & $0.541 \pm 0.058$ & $0.480 \pm_{0.009}^{0.008}$ & $3 / 4$ \\
\hline 2003 Sep 25 & $137.1 \pm 1.17$ & $0.376 \pm 0.031$ & $0.206 \pm_{0.006}^{0.017}$ & $3 / 2$ \\
\hline 2003 Oct 25 & $136.2 \pm 0.38$ & $0.589 \pm 0.030$ & $0.601 \pm_{0.001}^{0.020}$ & $2 / 3$ \\
\hline 2003 Dec 12 & $134.3 \pm 0.29$ & $0.518 \pm 0.062$ & $0.548 \pm_{0.001}^{0.028}$ & $2 / 2$ \\
\hline 2004 Jan 10 & $84.9 \pm 0.06$ & $0.248 \pm 0.013$ & $0.120 \pm_{0.001}^{0.007}$ & $2 / 2$ \\
\hline 2004 Jun 08 & $221.0 \pm 2.33$ & $0.478 \pm 0.068$ & $0.440 \pm_{0.032}^{0.020}$ & $2 / 2$ \\
\hline 2004 Dec 16 & $228.1 \pm 0.57$ & $0.534 \pm 0.069$ & $0.507 \pm_{0.014}^{0.006}$ & $3 / 3$ \\
\hline 2005 Feb 15 & $40.1 \pm 0.66$ & $0.468 \pm 0.064$ & $0.372 \pm_{0.011}^{0.009}$ & $2 / 5$ \\
\hline 2005 Dec 09 & $19.2 \pm 0.54$ & $0.505 \pm 0.075$ & $0.462 \pm_{0.012}^{0.006}$ & $2 / 3$ \\
\hline 2006 Jan 27 & $231.1 \pm 1.26$ & $0.500 \pm 0.063$ & $0.406 \pm_{0.009}^{0.016}$ & $2 / 3$ \\
\hline 2006 Apr 07 & $131.2 \pm 1.46$ & $0.466 \pm 0.052$ & $0.633 \pm_{0.045}^{0.010}$ & $3 / 2$ \\
\hline 2007 Jan 28 & $137.8 \pm 0.62$ & $0.362 \pm 0.039$ & $0.316 \pm_{0.009}^{0.026}$ & $3 / 2$ \\
\hline 2007 May 02 & $224.9 \pm 0.94$ & $0.453 \pm 0.065$ & $0.299 \pm_{0.006}^{0.028}$ & $2 / 2$ \\
\hline 2007 Oct 24 & $233.1 \pm 0.51$ & $0.363 \pm 0.029$ & $0.261 \pm_{0.010}^{0.007}$ & $2 / 1$ \\
\hline 2008 Jan 08 & $223.7 \pm 0.50$ & $0.249 \pm 0.029$ & $0.127 \pm_{0.008}^{0.009}$ & $4 y 5$ \\
\hline 2008 Jun 22 & $226.2 \pm 1.64$ & $0.401 \pm 0.032$ & $0.299 \pm_{0.006}^{0.010}$ & $4 / 2$ \\
\hline 2008 Jul 25 & $302.1 \pm 0.61$ & $0.343 \pm 0.025$ & $0.257 \pm_{0.007}^{0.016}$ & $2 / 2$ \\
\hline 2008 Sep 08 & $228.5 \pm 0.80$ & $0.323 \pm 0.023$ & $0.177 \pm_{0.008}^{0.025}$ & $1 / 2$ \\
\hline 2008 Sep 21 & $118.3 \pm 1.36$ & $0.384 \pm 0.025$ & $0.351 \pm_{0.010}^{0.031}$ & $2 / 2$ \\
\hline
\end{tabular}

Notes. The polar angle is the central angle of the cavity, measured counter-clockwise from the Sun's North pole. The Before/After column indicates the number of days the cavity was visible before and after the measurement date.

\section{REFERENCES}

Fisher, R. R., \& Poland, A. I. 1981, ApJ, 246, 1004

Fuller, J., Gibson, S. E., de Toma, G., \& Fan, Y. 2008, ApJ, 678, 515

Gibson, S. E., Fludra, A., Bagenal, F., Biesecker, D., Del Zanna, G., \& Bromage, B. 1999, J. Geophys. Res., 104, 9691

Gibson, S. E., Foster, D., Burkepile, J., de Toma, G., \& A., S. 2006, ApJ, 641, 590

Guhathakurta, M., Fludra, A., Gibson, S. E., Biesecker, D., \& Fisher, R. 1999, J. Geophys. Res., 104, 9801

Heinzel, P., et al. 2008, ApJ, 686, 1383

Hudson, H. S., Acton, L. W., Harvey, K. A., \& McKenzie, D. M. 1999, ApJ, 513,83

Hudson, H. S., \& Schwenn, R. 2000, Adv. Space Res., 25, 1859

Hundhausen., A. J. 1999, in The Many Faces of the Sun: A Summary of the Results of NASA's Solar Maximum Mission, ed. K. T. Strong, J. L. R. Saba, B. M. Haisch, \& J. T. Schmelz (New York: Springer), 143
Illing, R. M., \& Hundhausen, A. J. 1986, J. Geophys. Res., 91, 10951

Low, B. C., \& Berger, M. 2003, ApJ, 589, 644

Low, B. C., \& Wolfson, R. 1988, ApJ, 324, 574

Maricic, D., Vrsnak, B., Stanger, A. L., \& Veronig, A. 2004, Sol. Phys., 225, 337

Marqué, C. 2004, ApJ, 602, 1037

Marqué, C., Lantos, P., \& Delaboudinere, J.-P. 2002, A\&A, 387, 317

Sterling, A. C., \& Moore, R. L. 2004a, ApJ., 613, 1221

Sterling, A. C., \& Moore, R. L. 2004b, ApJ, 613, 1221

Titov, V. S., \& Demoulin, P. 1999, A\&A, 351, 707

van Ballegooijen, A. A., \& Cranmer, S. R. 2008, ApJ, 682, 644

Van de Hulst, H. C. 1950, BAAS, 11, 135

Vrsnak, B., Maricic, D., Stanger, A. L., \& Veronig, A. 2004, Sol. Phys., 225, 335

Yurchyshyn, V. B. 2002, AJ, 576, 493

Zhang, M., \& Low, B. C. 2004, ApJ, 600, 1043 